\title{
O CAMPO FORMAÇÃO DE PROFESSORES: UM ESTUDO QUANTITATIVO DOS ARTIGOS DA ÁREA DE EDUCAÇÃO MATEMÁTICA E DE ENSINO DE CIÊNCIAS NO BRASIL (1976 - 2007)
}

\author{
Angela Meneghello PASSOS ${ }^{1}$ \\ Marinez Meneghello PASSOS ${ }^{2}$ \\ Sergio de Mello ARRUDA ${ }^{3}$
}

\begin{abstract}
RESUMO: Este trabalho apresenta uma análise quantitativa de artigos sobre formação de professores publicados em periódicos da área de educação matemática e de ensino de ciências no Brasil (1976-2007). Os seguintes periódicos fizeram parte desta análise: Gepem - Boletim do grupo de estudos e pesquisas em Educação Matemática; Bolema - Boletim de Educação Matemática; Educação Matemática em Revista; Zetetiké; Educação Matemática Pesquisa; Revista Brasileira de Ensino de Física; Caderno Brasileiro de Ensino de Física; Ciência \& Educação; Investigações em Ensino de Ciências; Ensaio: pesquisa em educação em ciências; Revista Brasileira de Pesquisa em Educação em Ciências. Dos 3041 artigos analisados, 650 (21\%) pertencem ao campo formação de professores, sendo 474 da área de ensino de ciências e 176 artigos de educação matemática. Dentre os resultados obtidos, destacamos que, em geral, as revistas mais recentes, cujas publicações tiveram seu início nas décadas de 90 e no princípio deste século, são as que mais publicam sobre essa temática. Além disso, no cômputo geral, $67 \%$ do total de artigos em formação de professores estão concentrados nos últimos 8 anos (a partir de 2000).
\end{abstract}

PALAVRAS-CHAVE: Formação de professores. Análise quantitativa. Revistas de Educação Matemática. Revistas de Ensino de Ciências.

\section{Introdução}

Este trabalho faz parte de uma investigação que tem como objetivo geral uma compreensão histórica do campo ${ }^{4}$ formação de professores de matemática e de ciências, por meio das publicações em periódicos nacionais das áreas de educação matemática e de ensino de ciências. Os resultados desta investigação relativos à área de educação matemática estão em Passos (2009) e em Passos, Passos e Arruda (2009) apresentamos diversas considerações referentes à área de ensino de ciências.

\footnotetext{
${ }^{1}$ Mestranda em Ensino de Ciências e Educação Matemática. Universidade Estadual de Londrina. Centro de Ciências Exatas. Pós-Graduação em Ensino de Ciências e Educação Matemática.- Londrina - PR - Brasil. 86051-980 - angelamp@sercomtel.com.br

${ }^{2}$ UEL - Universidade Estadual de Londrina. Centro de Ciências Exatas - Departamento de Matemática. Londrina - PR - Brasil. 86051-980 - marinezmp@sercomtel.com.br

${ }^{3}$ UEL - Universidade Estadual de Londrina. Centro de Ciências Exatas - Departamento de Física. Londrina - PR - Brasil. 86051-980 - sergioarruda@sercomtel.com.br.(Com o apoio do CNPq).

${ }^{4}$ Adotamos o termo campo como “área em que se desenvolve certa atividade”, neste caso, pesquisas, reflexões e/ou projetos relativos à formação do professor.
} 
Para este momento trazemos nossas interpretações a respeito da leitura e das compreensões que tivemos ao estudar os dois corpus ${ }^{5}$ constituídos, independentemente a princípio, sendo que hoje buscamos uma relação e um movimento que possa se evidenciar deste trabalho conjunto.

Nossa primeira etapa foi a de realizar um levantamento da bibliografia, na forma de artigos em periódicos, disponíveis sobre a temática - formação do professor - no acervo constituído pelas onze revistas selecionadas para esse trabalho de pesquisa, indicando os percentuais relativos a cada acervo independentemente, bem como os relacionados a ambos. Destes procedimentos iniciais, cujos resultados podem ser observados em Passos (2009), verificamos por meio de um processo ancorado nos conceitos e desenvolvimentos apresentados pela teoria da análise textual que a formação de professores mostrava-se como uma tendência investigativa para a área de educação matemática. Promovemos, então, o desenvolvimento de uma proposta semelhante tendo como objetos de pesquisa periódicos da área de ensino de ciências - resultados semelhantes foram obtidos. Por esse motivo, justificamos hoje, neste trabalho nossa proposta de leitura integrada dos corpus constituídos para essas investigações cujas etapas preliminares já se esgotaram.

O que percebemos nas leituras primeiras deste material é que a formação do professor, como objeto de pesquisa, não se mostra, claramente, na indicação dos problemas ou das questões de pesquisa que pudemos observar nos artigos. Tivemos dificuldade para compreender se o que estava apresentado no artigo era objetivo ou questão geral de pesquisa. Por isso não fizemos distinção entre um e outro na constituição dos nossos corpus, pois muitos artigos confundiam objetivo com problema de pesquisa.

Para a escolha das revistas analisadas utilizamos o Sistema de Avaliação e Qualificação da Coordenação de Aperfeiçoamento de Pessoal de Nível Superior - Capes, o Qualis, e selecionamos os periódicos da área Ensino de Ciências e Matemática - voltados para a educação matemática e o ensino de ciências com avaliação níveis A ou B e circulação nacional (N) ou internacional (I) ${ }^{6}$.

Na sequência trazemos alguns dados quantitativos sobre o levantamento realizado e considerações que pudemos observar na leitura integrada das produções publicadas nesses periódicos. Em função do grande volume do material sob análise, o trabalho aqui descrito não pretende, nesta etapa da investigação, dar conta de todas as possibilidades, tornando-se

\footnotetext{
${ }^{5} \mathrm{O}$ conjunto dos documentos tidos em conta para serem submetidos aos procedimentos analíticos (BARDIN, 2004).

${ }^{6}$ Evidenciamos que durante o desenvolvimento da pesquisa esse sistema estava em processo de atualização, por isso usamos o Qualis vigente no ano de 2007.
} 
exaustivo, mas sim fornecer uma visão geral do que pudemos observar neste contato inicial com as revistas, desde suas primeiras edições. Em resumo, essas questões quantitativas, que aqui procuramos abordar, fazem parte de um programa de pesquisa cujo objetivo é aplicar as análises qualitativas - análise de conteúdo, análise de discurso, análise textual, análise textual discursiva - a investigações nas áreas de ensino de ciências e matemática, buscando contribuições desses referenciais para compreender alguns dos diversos campos pertinentes a essas áreas, entre eles, a formação do professor e a educação não formal, por meio da análise das produções bibliográficas em revistas qualificadas.

A seguir relacionamos o acervo constituído e algumas informações a respeito das revistas $^{7}$ que o compõem.

- Boletim do grupo de estudos e pesquisas em Educação Matemática - GEPEM. (51 revistas)

Trata-se de publicação do grupo de estudos e pesquisas em Educação Matemática Gepem - Instituto de Educação da Universidade Federal Rural do Rio de Janeiro ${ }^{8}$ RJ. Boletim n.1, 1976 / n.51, 2007.

- Boletim de Educação Matemática - Boletim do grupo de estudos e pesquisas em Educação Matemática - BOLEMA. (32 revistas)

Publicação do Instituto de Geociência e Ciências Exatas - Departamento de Matemática - Programa de pós-graduação em Educação Matemática - Unesp - Rio Claro - SP. n.1, Inverno de 1985 / n.28, 2007.

- Educação Matemática em Revista - EMR. (21 revistas)

Trata-se de uma das publicações da Sociedade Brasileira de Educação Matemática. n.1, 1993 / n.23, 2007.

- Zetetiké - ZETETIKÉ. (25 revistas)

Publicação do Círculo de estudo, memória e pesquisa em Educação Matemática da Faculdade de Educação da Universidade de Campinas. n.1, 1993 / n.28, 2007.

- Educação Matemática Pesquisa - EMP. (19 revistas)

Revista do Programa de estudos pós-graduados em Educação Matemática da Pontifícia Universidade Católica de São Paulo. v.1, n.1, 1999 / v.9, n.2, 2007.

- Revista Brasileira de Ensino de Física - RBEF. (81 revistas)

Publicação da Sociedade Brasileira de Física - São Paulo, SP. Antes do ano de 1992, respondia pelo nome - Revista de Ensino de Física. v.1, n.1, 1979 / v.29, n.4, 2007.

- Caderno Brasileiro de Ensino de Física - CBEF. (75 revistas)

Revista publicada pelo Departamento de Física do Centro de Ciências Físicas e Matemáticas da Universidade Federal de Santa Catarina - Florianópolis, SC. Informamos que antes do ano de 2002, este periódico era denominado por: Caderno Catarinense de Física. v.1, n.1, 1984 / v.24, n.3, 2007.

- Ciência \& Educação - CIEDU. (26 revistas)

Publicação da Universidade Estadual Paulista - Unesp - Faculdade de Ciências Programa de Pós-Graduação em Educação para a Ciência - Bauru - SP. Antes do ano

\footnotetext{
${ }^{7} \mathrm{~A}$ ordem em que as revistas são comentadas está relacionada à ordem cronológica em que foram editadas, isto é, da mais antiga para a mais recente, em cada acervo.

${ }^{8}$ Atualmente alocada neste instituto.
} 
de 1988, este periódico era intitulado - Série Ciência \& Educação. v.1, 1995 / v.13, n.3, 2007.

- Investigações em Ensino de Ciências - IENCI (36 revistas)

Revista publicada pelo Instituto de Física da Universidade Federal do Rio Grande do Sul - Porto Alegre, RS. v.1, n.1, 1996 / v.12, n.3, 2007.

- Ensaio: pesquisa em educação em ciências - ENSAIO (18 revistas)

Publicação do Centro de Ensino de Ciências e Matemática da Faculdade de Educação da Universidade Federal de Minas Gerais - Belo Horizonte, MG. v.1, n.1, 1999 / v.9, n.2, 2007.

- Revista Brasileira de Pesquisa em Educação em Ciências - RBPEC. (21 revistas)

- Publicação da Associação Brasileira de Pesquisa em Educação em Ciências - Abrapec - atualmente sediada em Belo Horizonte, MG. v.1, n.1, 2001 / v.7, n.3, 2007.

Das 405 revistas que compõe nosso acervo investigativo - 148, ou seja, 37\% são da área de educação matemática e 257, que equivalem percentualmente a 63\% do acervo, são da área de ensino de ciências. O período de edição em questão é de 32 anos - 1976/2007.

\section{O campo formação de professores: conceitos estruturantes, próximos e adjacentes ${ }^{9}$}

Como comentado nosso objetivo para o desenvolvimento desta pesquisa era o de identificar entre os inúmeros artigos publicados nesses periódicos aqueles que focavam ou tinham como temática de discussão, estudo ou reflexão, a formação de professores. Contudo restava-nos um dilema - como balizaríamos tais artigos, ou seja, quais seriam considerados pertinentes ao campo e quais não o seriam.

Os resultados desse movimento podem ser observados em Passos (2009) - naquela ocasião as palavras-chave dos artigos foram consideradas como disparadores para esses procedimentos seletivos, porém, para cada periódico tivemos que adequar nossa forma de trabalho pelo fato de que suas características editoriais nem sempre foram padronizadas e de periódico para periódico há algumas diferenças na estrutura e na composição dos elementos necessários para a publicação dos artigos.

Dessa etapa investigativa em que foram tomados para interpretação somente os periódicos da área de educação matemática e adotada como metodologia de coleta de dados e pesquisa a análise textual, tomando como teóricos Laurence Bardin (2004), Roque Moraes (1999, 2007), Pablo Navarro e Capitolina Díaz (1999), chegamos a uma listagem de 21 palavras que segundo nossas considerações encerravam em si a representatividade dos artigos do campo da formação de professores, ou seja, observando-as - as palavras-chave - era

\footnotetext{
${ }^{9}$ Adotamos tais adjetivos para os conceitos tendo como ponto de partida as discussões ocorridas no I Simpósio dos grupos de pesquisa sobre formação de professores no Brasil. Promoção do GT8 da Anped - 24 e 25 de julho de 2006 - Pontifícia Universidade Católica - SP.
} 
possível ter “uma idéia” sobre o que o artigo versava. Essa listagem em ordem alfabética, que também poderia ser distribuída em ordem cronológica - deu-nos uma visão temporal, sintética, compacta e focada do que passamos a considerar como relacionadas ao campo formação do professor:

- Currículo (1996), (2004); Desenvolvimento profissional (2001), (2004); Formação (2000), (2002); Formação continuada de professores (2003); Formação de professores (1996), (1997), (1998), (1999), (2000), (2001), (2002), (2003), (2004), (2005); Formação de professores contextualizada (2002); Formação de professores das séries iniciais (2004); Formação de professores de matemática (1997), (2003), (2004), (2005); Formação docente (2005); Formação inicial (1997), (2004); Formação inicial de professores (2003); Licenciatura (1999); Novas concepções pedagógicas (2001); Práticas (docentes) (1999); Professor de matemática (1999); Professor investigador (2003); Professor reflexivo (2001); Professores de matemática (2002); Reflexão sobre a prática (2005); Saber docente (2005); Teoria e prática (2002).

Diversos outros resultados e esclarecimentos sobre essas considerações dos últimos parágrafos deste trabalho podem ser observados em Passos (2009).

Um estudo pautado nessas etapas comentadas anteriormente foi iniciado, tomando como objeto analítico os periódicos da área de ensino de ciências. Além de uma relação de 112 palavras-chave, buscou-se evidenciar possíveis convergências entre as palavras dessa lista $^{10}$ - visivelmente alargada. Desse processo interpretativo e segundo algumas categorizações possibilitas pela análise de conteúdo concluiu-se que seria possível categorizar (organizar) a maioria delas em seis grupos principais, relacionados a:

1. Ação, ou seja: a ação didática, a atitude, a atividade, a atuação, o desempenho, o fazer, a intervenção, o exercício profissional; a prática (docente, profissional, instrucional, interdisciplinar, reflexiva, de ensino, cotidiana, de sala de aula, educacional, educativa, letiva e didático-pedagógica) - do professor, docente, educador ${ }^{11}$.

2. Formação, ou seja: o aperfeiçoamento, a aprendizagem, atualização, a autoformação, a capacitação, a educação, o estágio (supervisionado, de regência, pedagógico, curricular), a preparação, o treinamento; a formação (continuada, em serviço, inicial, básica, acadêmica, pedagógica, permanente, reflexiva) - de professores, pedagogos, profissionais, mestres, orientadores pedagógicos, etc.

3. Atributos (qualificações e/ou características subjetivas), tais como: a autonomia, a competência, os conflitos, a deficiência, a experiência, as falhas, a identidade, a mobilização, a motivação, o papel, o perfil, as preferências - dos professores.

4. Saber, que envolve: a compreensão, a concepção, o conhecimento (profissional, básico), as convicções, as crenças, o discurso, a experiência, a fala, a opinião, o

\footnotetext{
${ }^{10}$ A lista citada é comentada detalhadamente em Passos, Passos e Arruda (2009).

${ }^{11}$ Para fins de organização dos termos vamos considerar daqui para frente as palavras professor, docente e educador como sinônimos.
} 
pensamento, a percepção, as perspectivas, a reflexão, as representações, a visão - de professores, futuros professores ou do corpo docente.

5. Profissão, ou seja: a carreira, função, o ofício, a tarefa, o trabalho - do professor.

6. Identidade, ou seja, do professor, enquanto: formador, investigador, pesquisador, reflexivo; estagiário, aluno; em exercício, em formação, em serviço, em treinamento; do Ensino Médio, Ensino Fundamental, $1^{\circ}$ grau, $2^{\circ}$ grau, séries iniciais, universitário, EJA, Ensino Básico, nível secundário e terciário, ensino primário e secundário; de Matemática, Física, Biologia, Geologia, Ciências, Ciências Naturais, Ciências da Natureza, Química, Ciências Biológicas, Ecologia, História, Redação.

A partir dessas interpretações passamos a assumir em nosso contexto investigativo que esses seis itens podem caracterizar, de forma mais resumida, a área de formação de professores de matemática e de ciências como sendo aquela que se refere: à ação do professor; à sua formação; aos seus atributos, qualificações e características subjetivas; ao seu saber; à sua profissão; e à identidade do professor.

\section{Constituição, quantificação do corpus e algumas considerações}

Cabe lembrar que a pesquisa aqui exposta está se desenvolvendo de forma gradativa. Nesse momento, estamos nos dedicando a um trabalho de caráter quantitativo, estruturado em leituras e releituras do acervo selecionado, segundo o desenvolvimento metodológico apresentado na seção anterior. Os critérios estabelecidos nesta etapa que foi deflagrada tendo como disparadores as palavras-chave e culminou em um processo de categorização e convergência para 6 itens - ação, formação, atributos, saber, profissão e identidade - nos possibilitou a constituição de um corpus cujos dados quantitativos apresentamos na sequência.

No Quadro 1 apresentamos a quantidade de artigos de cada revista analisada e a quantidade de artigos que identificamos, interpretamos e assumimos - segundo nossos critérios analíticos - que sejam do campo da formação de professores.

\begin{tabular}{|c|c|c|c|c|}
\hline Área & $\begin{array}{c}\text { Sigla referente ao } \\
\text { nome da revista }\end{array}$ & $\begin{array}{c}\text { Total de } \\
\text { artigos }\end{array}$ & $\begin{array}{c}\text { Total de artigos } \\
\text { sobre formação } \\
\text { de professores }\end{array}$ & $\begin{array}{c}\text { Percentagem } \\
\text { artigos referentes à } \\
\text { formação de } \\
\text { professores }\end{array}$ \\
\hline \multirow{3}{*}{ Educação } & ZETETIKÉ & 143 & 50 & $40 \%$ \\
\cline { 2 - 5 } matemática & EMR & 172 & 45 & $26 \%$ \\
\cline { 2 - 5 } & EMP & 76 & 12 & $16 \%$ \\
\cline { 2 - 5 } & GEPEM & 309 & 42 & $14 \%$ \\
\hline
\end{tabular}

\footnotetext{
${ }^{12}$ Destacamos que as porcentagens indicadas são aproximadas.
} 


\begin{tabular}{|c|c|c|c|c|}
\hline & BOLEMA & 202 & 27 & $13 \%$ \\
\hline \multirow{3}{*}{$\begin{array}{c}\text { Ensino de } \\
\text { ciências }\end{array}$} & ENSAIO & 102 & 48 & $47 \%$ \\
\cline { 2 - 5 } & RBPEC & 137 & 62 & $45 \%$ \\
\cline { 2 - 5 } & IENCI & 163 & 64 & $39 \%$ \\
\cline { 2 - 5 } & CIEDU & 264 & 88 & $33 \%$ \\
\cline { 2 - 5 } & CBEF & 453 & 80 & $18 \%$ \\
\cline { 2 - 5 } & RBEF & 1020 & 132 & $13 \%$ \\
\cline { 2 - 5 } & TOTAL & $\mathbf{3 0 4 1}$ & $\mathbf{6 5 0}$ & $\mathbf{2 1 \%}$ \\
\hline
\end{tabular}

Quadro 1- Quantidade de artigos das revistas

De acordo com o quadro anterior, a quantidade total de artigos encontrados nas revistas analisadas é de 3041 artigos - 2139 em revistas da área de ensino de ciências, representando um percentual de, aproximadamente, 70\%; 902 em revistas da área de educação matemática, totalizando 30\% dos artigos que compõem o material analisado para esta pesquisa. Quanto aos identificados como pertencentes ao campo formação de professores, esses somam 650, sendo que 474 (73\%) foram publicados em revistas da área de ensino de ciências e os demais - 176 artigos (27\%) - encontram-se em periódicos da área de educação matemática.

Ao observarmos cada área independentemente e relacionarmos os artigos publicados e a percentagem que representam aqueles que se debruçam sobre pesquisas, ensaios e/ou reflexões, sobre a formação de professores temos que: para a área de ensino de ciências - dos 2139 artigos, 474 focam a temática formação do professor, representando 22\% dos artigos pesquisados, avaliados e selecionados desses periódicos; para a área de educação matemática - dos 902 artigos, 176 referem-se a desenvolvimentos no campo da formação de professores de matemática, perfazendo um percentual de, aproximadamente 20\%, dos artigos publicados. Esses resultados nos mostram que, independentemente da área de trabalho e de publicação para essa análise que se limita às áreas de educação matemática e ensino de ciências - há uma constância nos percentuais de publicação de artigos relacionados ao campo de pesquisa analisado - ou seja, em ambas as áreas cerca de $21 \%$ dos artigos publicados são resultados de pesquisas, reflexões, intervenções, ensaios ou propostas que possuem a formação de professores como objeto de estudo.

Ao observarmos cada revista, em suas respectivas áreas, podemos verificar também que, em geral, as revistas mais recentes, cujas publicações tiveram seu início nas décadas de 90 e no princípio deste século - são as que mais publicam sobre essa temática: Zetetiké (40\%) datada de 1993; EMR (26\%) datada de 1993; EMP (16\%) datada de 1999; Ensaio (47\%) datada de 1999; RBPEC (45\%) datada de 2001; IENCI (39\%) datada de 1996; CIEDU (33\%) 
datada de 1995, em contraposição a aquelas que tiveram suas publicações iniciadas nas décadas de 70 e 80 do século passado.

No Quadro 2 é possível observar um levantamento ano a ano da quantidade de artigos sobre formação de professores nas revistas selecionadas das áreas de educação matemática e ensino de ciências.

\begin{tabular}{|c|c|c|}
\hline Ano & $\begin{array}{l}\text { Total de artigos nas revistas da } \\
\text { área de educação matemática }\end{array}$ & $\begin{array}{l}\text { Total de artigos nas revistas da } \\
\text { área de ensino de ciências }\end{array}$ \\
\hline 1976 & $\mathbf{0}$ & \\
\hline 1977 & $\mathbf{0}$ & \\
\hline 1978 & $\mathbf{0}$ & \\
\hline 1979 & $\mathbf{0}$ & 4 \\
\hline 1980 & 3 & 9 \\
\hline 1981 & 1 & 5 \\
\hline 1982 & $\mathbf{0}$ & 2 \\
\hline 1983 & 1 & 3 \\
\hline 1984 & 1 & 3 \\
\hline 1985 & 2 & 3 \\
\hline 1986 & $\mathbf{0}$ & 5 \\
\hline 1987 & 2 & 3 \\
\hline 1988 & $\mathbf{0}$ & 6 \\
\hline 1989 & 2 & 6 \\
\hline 1990 & 3 & 8 \\
\hline 1991 & 2 & 7 \\
\hline 1992 & 1 & 34 \\
\hline 1993 & 2 & 4 \\
\hline 1994 & 3 & 4 \\
\hline 1995 & 4 & 4 \\
\hline 1996 & 8 & 13 \\
\hline 1997 & 9 & 7 \\
\hline 1998 & 3 & 16 \\
\hline 1999 & 9 & 17 \\
\hline 2000 & 6 & 24 \\
\hline 2001 & 16 & 46 \\
\hline 2002 & 22 & 32 \\
\hline 2003 & 11 & 33 \\
\hline 2004 & 12 & 45 \\
\hline 2005 & 13 & 49 \\
\hline 2006 & 17 & 35 \\
\hline 2007 & 23 & 47 \\
\hline Totais & 176 & 474 \\
\hline
\end{tabular}

Quadro 2- Levantamento ano a ano da quantidade de artigos sobre formação de professores nas revistas das áreas de educação matemática e ensino de ciências.

Observação: As células em branco, no quadro, referem-se aos anos em que os periódicos não eram editados.

Observando o Quadro 2 é possível verificar que a primeira publicação sobre a temática formação do professor na área de educação matemática é datada de 1980, sendo que o periódico mais antigo é do ano de 1976. Referente a essa mesma questão observa-se, que, 
nos periódicos da área de ensino de ciências, há a presença desta temática desde os primeiros números - datados de 1979.

Os dados também revelam que a partir de 2000 a incidência desse tema aumentou consideravelmente nas duas áreas. No cômputo geral, 67\% do total de artigos em formação de professores estão concentrados nos últimos 8 anos (a partir de 2000). Destacamos que as percentagens das áreas para esse período são muito próximas - educação matemática 68\% e ensino de ciências 66\%, respeitando a proporcionalidade de artigos publicados em cada uma das áreas. Esses fatos também podem ser visualizados de forma mais evidente no gráfico a seguir.

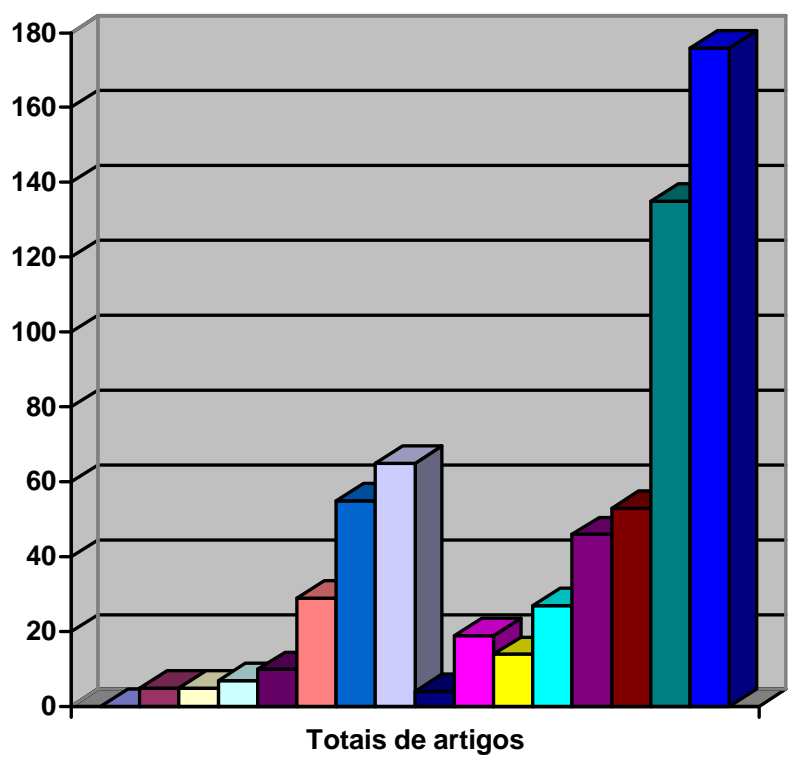

\begin{tabular}{|c|}
\hline$\square$ EM 1976-1979 \\
\hline$\square$ EM 1980-1983 \\
\hline$\square$ EM 1984-1987 \\
\hline 口EM 1988-1991 \\
\hline 口EM 1992-1995 \\
\hline 口EM 1996-1999 \\
\hline 口EM 2000-2003 \\
\hline 口EM 2004-2007 \\
\hline DEC 1979 \\
\hline 口EC 1980-1983 \\
\hline 口EC 1984-1987 \\
\hline EC 1988-1991 \\
\hline 口EC1992-1995 \\
\hline 口EC 1996-1999 \\
\hline 口EC 2000-2003 \\
\hline EC 2004-2007 \\
\hline
\end{tabular}

Gráfico 1 - As publicações, em periódicos da área de Educação Matemática (EM) e da área de Ensino de Ciências (EC), sobre formação de professores em 32 anos (1976 a 2007) ${ }^{13}$.

\footnotetext{
${ }^{13}$ Construímos o gráfico em intervalos de quadriênios. Para acomodar as publicações da área de ensino de ciências em quadriênios, o ano de 1979 ficou sozinho em nossa representação.
} 
Ao observarmos o gráfico evidencia-se com mais clareza o que o quadro anterior nos informava, por exemplo, a quantidade de artigos sobre formação de professores publicados pela área de ensino de ciências é bem maior do que os artigos publicados pela área de educação matemática - 73\% dos 650 selecionados - segundo nossos critérios analíticos.

O gráfico também nos mostra o movimento dessas duas áreas - afora as quantidades diferenciadas dos artigos publicados - no que diz respeito à tendência de publicar resultados de pesquisa, reflexões, intervenções e/ou ensaios, referentes ao campo da formação de professores. Nos dois conjuntos de colunas basta focarmos as três últimas de cada bloco em comparação com as iniciais, respectivamente.

Mesmo sendo áreas distintas, as tendências em pesquisar ou refletir sobre a formação de professores, apresenta-se em movimento aproximados no que diz respeito ao período de início e continuidade dessas pesquisas e à percentagem crescente - cada uma dentro de suas proporcionalidades.

\section{Considerações Finais}

Nessas considerações finais, primeiramente, retomamos a questão maior que deflagrou esse levantamento e que compõem o programa de pesquisa de nosso grupo: quais os sentidos de formação docente que estão subjacentes ou explícitos nos artigos publicados nesses periódicos das áreas de educação matemática e de ensino de ciências?

Para buscar uma compreensão do que pretendíamos responder, algumas estratégias orientaram nossas ações - entre elas, a constituição do acervo; a seleção dos artigos; as leituras preliminares desses artigos; a opção em assumir que as palavras-chave encerram em si o significado global de um contexto; a constituição e definição de unidades de pesquisa e de análise.

Como indicado no início deste trabalho, nos propusemos a trazer para este evento as quantificações do que observamos - fase preliminar, mas concluída - de uma pesquisa que se encontra em desenvolvimento e que se estrutura sobre os dados quantitativos desta etapa já encerrada que aqui apresentamos.

Essas três décadas de pesquisa evidenciadas nesses periódicos podem ser assumidas como um documento que apresenta possibilidades para a formação inicial e contínua de professores, pois traz informações que permitem a aquisição de novos saberes e a ação relativa a novas experiências docentes, possibilitando o renovar e um novo significado para a prática e os saberes do professor. 
Foi também possível identificar que muito se pesquisa sobre o campo formação de professores no Brasil e que o nosso movimento "nas” e “das” pesquisas mostra-se em paralelo ao que se desenvolve em âmbito internacional - considerando, inclusive, nossas especificidades e particularidades. E que a ênfase dada aos teóricos e referenciais estrangeiros é muito maior do que o destaque ao que é produzido e elaborado no país.

Quanto aos critérios de seleção dos artigos utilizados por nós, estamos cientes de que (esses critérios - assumidos e adotados) podem ter negligenciado ou deixado à margem ou de fora alguns artigos, entretanto, cremos que os que foram selecionados contribuíram com a compreensão do que buscávamos. E nos mostraram um movimento das áreas com relação ao campo da formação de professores.

Além disso, neste momento de encerramento, gostaríamos de destacar que há poucos periódicos da área de educação matemática no Brasil e que muitos artigos produzidos não foram neles publicados por fatores como periodicidade; quantidade reduzida de artigos por periódicos; por serem poucos para uma comunidade de pesquisadores crescente; por se manterem os mesmos por mais de décadas, ou seja, não surgiram novos periódicos nos últimos anos apesar de a produção de pesquisas ter aumentado significativamente, a quantidade de doutores no Brasil ter crescido substancialmente na última década (informações da mídia, dos órgãos públicos e de fomento) - como todos sabem. Fato esse que para a área de ensino de ciências em nosso país não tem a mesma constatação.

Levantamos, no tocante às problemáticas, que a educação matemática encontra-se em uma interface e tem também espaço de publicação junto à Educação, e os periódicos dessa área não foram, nesta investigação, acessados para estudo e análise.

Para este parágrafo final, nos possibilitamos uma visão "macro” do que toda essa caminhada e construção nos mostrou - "se somos fruto do que vivemos na escola" quem sabe a situação e o estado em que a escola se encontra hoje mude em breve, principal e fundamentalmente, por estarmos buscando novas opções para essa escola por meio do estudo sobre a formação dos professores. 


\title{
THE FIELD OF TEACHERS EDUCATION: A QUANTITATIVE STUDY OF PAPERS OF MATHEMATICS AND SCIENCE EDUCATION AREAS IN BRAZIL (1976 - 2007)
}

\begin{abstract}
This work presents a quantitative analysis of papers on teacher education published in journals of mathematics and science education areas in Brazil (1976-2007). We analysed the following journals: Gepem - Boletim do grupo de estudos e pesquisas em Educação Matemática; Bolema - Boletim de Educação Matemática; Educação Matemática em Revista; Zetetiké; Educação Matemática Pesquisa; Revista Brasileira de Ensino de Física; Caderno Brasileiro de Ensino de Física; Ciência \& Educação; Investigações em Ensino de Ciências; Ensaio: pesquisa em educação em ciências; Revista Brasileira de Pesquisa em Educação em Ciências. Of the 3041 articles examined, 650 (21\%) belong to teachers education field, being 474 of science education and 176 of mathematics education. Among the results, we highlight that, in general, the journals dated from the 90s and the beginning of 00's decade, are those that publish more on this topic. Furthermore, 67\% of all articles in teachers education published in the two areas were found in the last 8 years (from 2000).
\end{abstract}

KEYWORDS: Teacher education. Quantitative analysis. Journals of Mathematics Education. Journals of Science Education.

\section{REFERÊNCIAS}

BARDIN, L. Análise de conteúdo. 4.ed. Tradução de Luís Antero Reto e Augusto Pinheiro Lisboa: Edições 70, 2004.

MORAES, R. Análise de conteúdo. Educação, Porto Alegre, v.22, n.37, p.7-32, mar. 1999.

MORAES, R.; GALIAZZI, M. C. Análise textual discursiva. Ijuí: Ed. da Unijuí, 2007.

NAVARRO, P.; DÍAZ, C. Análisis de contenido. In: DELGADO, J. M.; GUTIÉRREZ, J. (Coord.) Métodos y técnicas cualitativas de investigación en ciencias sociales. Madrid: Síntesis, 1999. p.177-224.

PASSOS, A. M.; PASSOS, M. M.; ARRUDA, S. de M. Caracterização do campo formação de professores por meio da análise quantitativa de artigos da área de Ensino de Ciências no Brasil. [S.l.], 2009. No prelo. Artigo em submissão.

PASSOS, M. M. O professor de matemática e sua formação: análise de três décadas da produção bibliográfica em periódicos na área de Educação Matemática no Brasil. 2009. 328 f. Tese (Doutorado em Educação) - Faculdade de Ciências, Universidade Estadual Paulista, Bauru, 2009. 